\title{
Dynamics of periodic impulsive collision in escapement mechanism
}

\author{
Jian $\mathrm{Mao}^{\mathrm{a}, *}, \mathrm{Yu} \mathrm{Fu}^{\mathrm{b}}$ and Peichao $\mathrm{Li}^{\mathrm{a}}$ \\ a Shanghai University of Engineering Science, Shanghai, China \\ ${ }^{\mathrm{b}}$ Tianjin Seagull Watch Co. Ltd, Tianjin, China
}

Received 10 May 2012

Revised 25 January 2013

Accepted 13 April 2013

\begin{abstract}
Among various non-smooth dynamic systems, the periodically forced oscillation system with impact is perhaps the most common in engineering applications. The dynamical study becomes complicated due to the impact. This paper presents a systematic study on the periodically forced oscillation system with impact. A simplified model of the escapement mechanism is introduced. Impulsive differential equation and Poincare map are applied to describe the model and study the stability of the system. Numerical examples are given and the results show that the model is highly accurate in describing/predicting their dynamics.
\end{abstract}

Keywords: Periodic collision, dynamics, escapement mechanism

\section{Introduction}

Dynamics is an important branch of mechanical engineering. In the last century, the linear dynamics was well studied and understood. However, nonlinear dynamics is far from being fully understood because of its complexity and diversity [1]. Non-smooth dynamical system is a special category of nonlinear system. In practice, it is not difficult to find non-smooth dynamical systems, such as a hammer hitting a nail, a signal triggering an electrical circuit, and a disaster affecting the stock market.

It is known that a typical smooth dynamical system can be described by an autonomous set of Ordinary Differential Equations (ODEs) [2-6]. Newton method and Lagrange's equation are two basic tools to model such dynamical systems. However, at present there is no universal method to model and solve non-smooth dynamical system. Various tools were developed in the last half century, including differential inclusion, Moreau's sweeping process, linear complementarity method and impulsive differential equations. Each of them only works for some cases.

Nonlinear oscillation is one category of periodical dynamic system with nonlinearity. The nonlinearity may be caused by phenomena such as contact, friction and impact. Guckenheimer [7] develops a method to describe the property of such motion. Fidlin [8] gives a systematic description of nonlinear oscillation in mechanical system. Change of parameter will result in differential behavior, either converging to a limit cycle or turning into chaos. The bifurcation theory is therefore developed. A special sub-category, called vibro-impact system which involves periodic impacts, draws a good amount of attention. Shaw and Holmes [9] study a periodically forced single degree of freedom non-linear oscillator and present a framework to solve the bifurcation problem. Peterka [10] introduced a

${ }^{*}$ Corresponding author: Jian Mao, Shanghai University of Engineering Science, Shanghai 201620, China. E-mail: jmao@sues.edu.cn. 


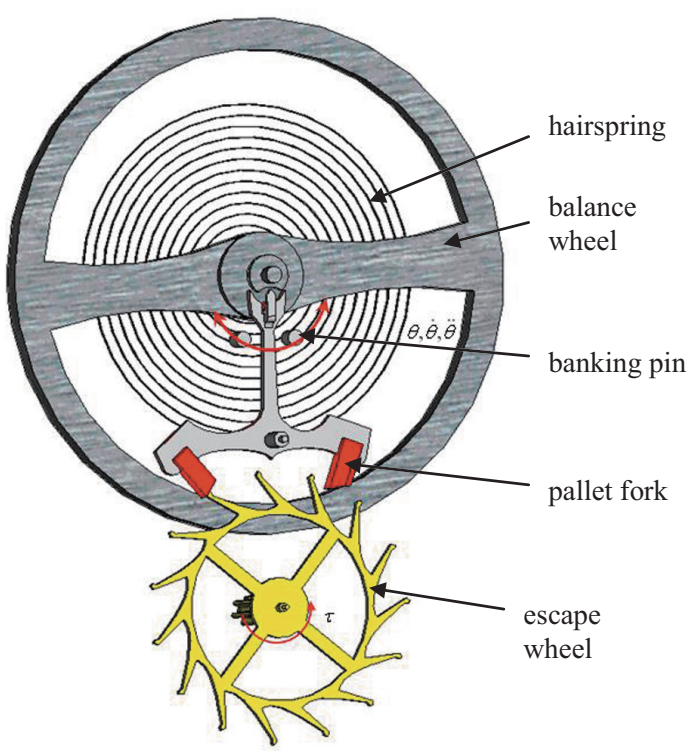

Fig. 1. Illustration of the Swiss lever escapement.

double impact oscillator. The anti-phase impact motion has dynamics identical to those of the single system. The inphase motion and the influence of asymmetries of the system parameters are studied through numerical simulations. Janin and Lamarque [11] studied the stability problem of a single DOF vibro-impact oscillator.

The vibro-impact system has lots of applications. Zhang et al. [12] presents a high frequency MEMS switch. In this system, a micro-fabricated cantilever beam repeatedly hits other structure and controls the circuit with a high frequency. Clock escapement [13-15] has the similar feature, which is related to our case study but less complicated.

In this paper, a new approach is presented to analyze the periodically forced oscillation system with impact. In Sections 2, the escapement mechanism is introduced and the theoretical model is developed. The nonlinear oscillation is studied in Section 3, including the solution and stability analysis. The numerical examples are given in Section 4. Finally, the conclusions are drawn in Section 5.

\section{System model}

Mechanical watch movement is one of the oldest mechanisms. Among all the components, the escapement is the most important because it controls the running speed of the watch. Though the working principle has been well understood, little research about the dynamics of the mechanism can be found.

Figure 1 shows the Swiss lever escapement mechanism, which is the most common escapement in current watch movements. It consists of five components: a balance wheel, a hairspring, two banking pins, a pallet fork and an escape wheel. Note that one end of the hairspring is fixed while the other end is attached to the balance wheel. The balance wheel oscillates periodically under the restoring force of the hairspring. The driving force from the escape wheel through the pallet fork compensates the consuming energy during oscillation. The guard pin, which is a synthetic ruby on the balance wheel, sends and receives impulses from the pallet fork to the balance wheel. The banking pin limits the rotation of the pallet fork. The escape wheel rotates intermittently at a certain speed according to the frequency of the system. This frequency is mainly determined by the property of balance wheel and hairspring. However, the value changes due to the existence of external force and impact.

The escapement can be simplified to a system as shown in Fig. 2. In the first half cycle, the mass move from left to right. Collision occurs at position $a_{1}$. The force $F(t)$ acts in the same direction from position $a_{1}$ to position $a_{2}$. In the second half cycle, the mass moves from right to left. Collision occurs at position $a_{3}$. The force $F(t)$ acts in the same direction from position $a_{3}$ to position $a_{4}$. 


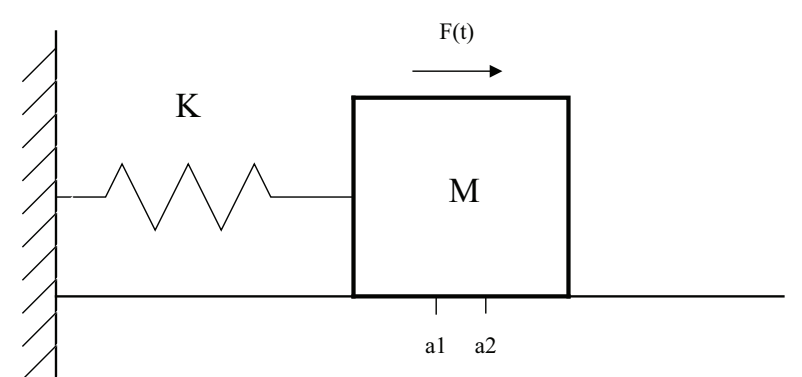

(a) First half cycle

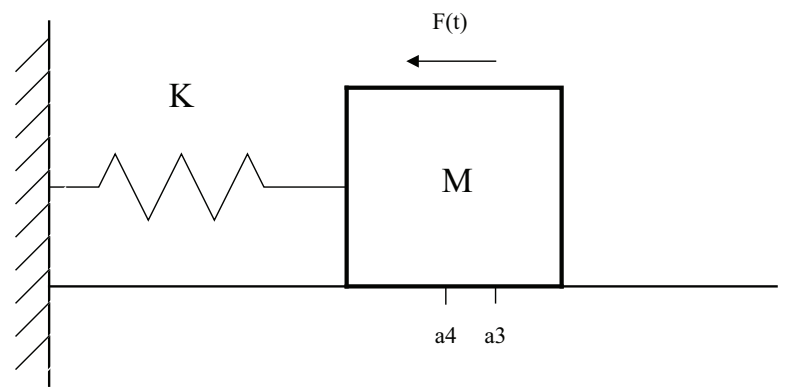

(b) Second half cycle

Fig. 2. Schematic diagram of the vibro-impact system.

\section{The dynamics of the model}

\subsection{Impulsive differential equation}

In order to study the dynamics of the escapement, the impulsive differential equation is applied. There are three components in an impulsive differential equation: a continuous-time-differential equation, an impulse equation and a jump criterion. The first one determines the states between impulses. The second describes the jump by a jump function at the instant of the impulse. The last one is an event set by which the jump function works.

Let $\Omega$ be the phase space of some evolutionary process, i.e., the set of its states, then these three components can be written by a general differential equation as follows [16]:

$$
\begin{cases}\frac{d x}{d t}=f(t, x), & \text { if } \phi(x) \neq 0 \\ \Delta x=\rho(x), & \text { if } \phi(x)=0 \\ x\left(t_{0}^{+}\right)=x_{0} & \end{cases}
$$

where, $f: R \times \Omega \rightarrow R^{n},\{x \mid \Phi(x)=0\}$ is the jump set, $\rho: R \times \Omega \rightarrow \Omega$ is the jump function.

\subsection{Poincare map}

The stability of a periodic orbit of the original system is closely related to the stability of the fixed point of the corresponding Poincare map [9]. Poincare map is the intersection of a periodic orbit in the state space of a continuous dynamical system with a certain lower dimensional subspace, called the Poincare section. More precisely, one considers a periodic orbit with initial conditions on the Poincare section and observes the points at which this orbit returns to the section. A Poincare map can be interpreted as a discrete dynamical system with a state space that has one fewer dimension than the original continuous dynamical system. Because it preserves many properties of periodic and quasiperiodic orbits of the original system and has a lower dimensional state space it is often used for analyzing the original system.

\subsection{Solution of the system}

The simplified escapement system can be described using the impulsive differential equations as follows,

$$
\begin{cases}\frac{d y}{d t}=A(t) y+g(t), & \text { if } \phi\left(y\left(\tau_{k}\right)\right) \neq 0 \\ \Delta y=B y, & \text { if } \phi\left(y\left(\tau_{k}\right)\right)=0\end{cases}
$$

with the initial condition $y\left(t_{0}^{+}\right)=y_{0}$. Here $y$ is the state defined by $y \in[x \dot{x}]^{T} . A(t), g(t)$ are functions of time $t$. $\tau_{k}$ is the instance where the impact occurs, $k=1,2, \ldots$ 
It is a linear non-homogeneous impulsive system. Each solution $y=\varphi(t)$ which satisfies the condition $\varphi\left(t_{0}^{+}\right)=$ $y_{0}$ is a solution of the initial value problem [16]. The motion can be described in eight different steps. The derivation of the system equation for each step can be found in Appendix A. After modeling for each step, the matrix can be found out as follows:

$$
\begin{aligned}
& A=\left[\begin{array}{cc}
0 & 1 \\
-\omega_{n} & 0
\end{array}\right] \\
& g(t)=\left\{\begin{array}{l}
{\left[\begin{array}{l}
0 \\
0
\end{array}\right] \quad x \in S_{1}} \\
{\left[\begin{array}{c}
0 \\
\sigma f
\end{array}\right] x \in S_{2}}
\end{array}\right. \\
& B=\left[\begin{array}{cc}
0 & 0 \\
0 & -b
\end{array}\right]
\end{aligned}
$$

where

$\omega_{n}^{2}=k=\frac{K}{M}$ is the natural frequency, $K$ is the spring constant and $M$ is the mass

$\sigma= \begin{cases}+1, & 1 \text { st half period } \\ -1, & \text { 2nd half period }\end{cases}$

$f=\frac{F}{M}$ and $F$ is the force,

$b$ satisfies $\Delta \dot{x}=-b \dot{x}$.

Let $S$ be the hypersurface of $\phi\left(y\left(\tau_{k}\right)\right)=0$,

$$
S=\left\{y: x \in\left\{a_{1}, a_{3}\right\}, x \cdot \dot{x}<0\right\}
$$

The supplementary set of the jump set is $\bar{S}=S_{1} \cup S_{2}$, where

$$
\left\{\begin{array}{l}
S_{1}=\left\{y: x<a_{1}, \dot{x}>0\right\} \cup\left\{y: x>a_{2}, \dot{x}>0\right\} \cup\left\{y: x>a_{3}, \dot{x}<0\right\} \cup\left\{y: x<a_{4}, \dot{x}<0\right\} \\
S_{2}=\left\{y: x \in\left(a_{1}, a_{2}\right), \dot{x}>0\right\} \cup\left\{y: x \in\left(a_{4}, a_{3}\right), \dot{x}<0\right\}
\end{array}\right.
$$

Because $A$ and $g(t)$ are Lipschitz continuous in a neighborhood of $\left(t_{0}, y_{0}\right)$, the initial value problem has exactly one solution [16].

\subsection{The stability}

The stability of impulsive system can be determined by the following theorem [17].

For an autonomous impulsive equation,

$$
\begin{cases}\frac{d x}{d t}=f(x, \varepsilon), & \text { if } x \notin \sigma(\varepsilon) \\ \Delta x=g(x), & \text { if } x \in \sigma(\varepsilon)\end{cases}
$$

where $a ̊$ is a small parameter, with $\stackrel{\AA}{a}=0$. Let the Floquet multipliers $\grave{i}_{j}$ satisfy $\mu_{1}=1,\left|\grave{i}_{j}\right|<1$, then the $T_{0}$-periodic solution $x=\varphi(t)$ with $\varepsilon=0$ is orbitally asymptotically stable.

Because the multipliers are also the eigenvalues of the Poincare map, they can be found out in an easier way.

In the escapement model $y$ is a 2-dimensional state. Define the Poincare map

$$
P: \Sigma \rightarrow \Sigma
$$


where, $\Sigma$ is Poincare section defined by,

$$
\Sigma=\left\{y \mid y \in R^{2}, y_{1}=a_{1}, y_{2}>0\right\}
$$

Each Poincare section indicates the state in which the mass passes a certain position with certain velocity direction in each cycle. In this system each cycle only contains one such state.

We can obtain

$$
y(k+1)=\left(\begin{array}{c}
a_{1} \\
\dot{x}(k+1)
\end{array}\right)=P(y(n))=\left(\begin{array}{c}
a_{1} \\
g(\dot{x}(k))
\end{array}\right)
$$

Therefore $\mu_{1}=1$.

Denoted by $t(k), x(k), \dot{x}(k), h(k)$ are the related instant, displacement, velocity and amplitude in the $k$ th cycle. According to the analysis in Appendix A, one cycle of the motion can be divided into eight steps. $t_{0}(k), t_{1}(k), t_{2}(k)$, $t_{1}(k), t_{3}(k), t_{4}(k)$, and $t_{5}(k)$, are the starting instant of the 1st, 2nd, 4th, 5th, 6th, 8th step respectively. $t_{6}(k)$ is the ending instant of the 8th step. $h_{0}(k), h_{1}(k), h_{2}(k), h_{3}(k)$ and $h_{4}(k)$ are the amplitude of the $1 \mathrm{st}, 3 \mathrm{rd}, 4 \mathrm{th}, 7 \mathrm{th}, 8 \mathrm{th}$ step respectively. Then,

$$
\begin{aligned}
& t_{0}(k+1)=t_{6}(k) \\
& h_{0}(k+1)=h_{4}(k)
\end{aligned}
$$

At instant $t_{1}(k)$, the velocity after impact is

$$
\dot{x}_{1}^{+}(k)=(1-b) \dot{x}_{1}(k)
$$

At instant $t_{2}(k)$, the velocity can be obtained by solving the Eq. (A-3)

$$
\dot{x}_{2}(k)=\sqrt{\left(\dot{x}_{1}^{+}(k)\right)^{2}+\left(a_{1}^{2}-a_{2}^{2}\right) \omega_{n}^{2}-2\left(a_{1}-a_{2}\right) f}
$$

At instant $t_{4}(k)$, the velocity can be obtained by solving the Eq. (A-1)

$$
\dot{x}_{4}(k)=-\sqrt{\left(\dot{x}_{2}(k)\right)^{2}+\left(a_{2}^{2}-a_{3}^{2}\right) \omega_{n}^{2}}
$$

At instant $t_{4}(k)$, the velocity after impact is

$$
\dot{x}_{4}^{+}(k)=(1-b) \dot{x}_{4}(k)
$$

At instant $t_{5}(k)$, the velocity can be obtained by solving the Eq. (A-5)

$$
\dot{x}_{5}(k)=-\sqrt{\left(\dot{x}_{4}^{+}(k)\right)^{2}+\left(a_{3}^{2}-a_{4}^{2}\right) \omega_{n}^{2}-2\left(a_{3}-a_{4}\right) f}
$$

At instant $t_{1}(k+1)$, the velocity can be obtained by solving the Eq. (A-5)

$$
\dot{x}_{1}(k+1)=\sqrt{\left(\dot{x}_{5}(k)\right)^{2}+\left(a_{4}^{2}-a_{1}^{2}\right) \omega_{n}^{2}}
$$

Substituting Eqs (10) (14) into Eq. (15), we get

$$
\begin{aligned}
\dot{x}_{1}(k+1) & =g\left(\dot{x}_{1}(k)\right) \\
& =\sqrt{(1-b)^{4} \dot{x}_{1}^{2}(k)+\left(b^{2}-2 b\right) \omega_{n}^{2}\left(a_{1}^{2}-a_{3}^{2}\right)-2(1-b)^{2}\left(a_{1}-a_{2}\right) f+2\left(a_{3}-a_{4}\right) f}
\end{aligned}
$$


Assuming $a_{1}=-a_{3}, a_{2}=-a_{4}$, we get

$$
\dot{x}_{1}(k+1)=g\left(\dot{x}_{1}(k)\right)=\sqrt{(1-b)^{4} \dot{x}_{1}^{2}(k)+2\left(b^{2}-2 b+2\right)\left(a_{2}-a_{1}\right) f}
$$

Let $\dot{x}_{1}^{*}$ be a fixed point, there is

$$
g\left(\dot{x}_{1}^{*}\right)=\dot{x}_{1}^{*}
$$

The solution is

$$
\dot{x}_{1}^{*}=\sqrt{\frac{2\left(a_{2}-a_{1}\right) f}{2 b-b^{2}}}
$$

The derivative of Eq. (17) is

$$
g^{\prime}\left(\dot{x}_{1}(k)\right)=\frac{(1-b)^{4} \dot{x}_{1}(k)}{\sqrt{(1-b)^{4} \dot{x}_{1}^{2}(k)+2\left(b^{2}-2 b+2\right)\left(a_{2}-a_{1}\right) f}}
$$

The eigenvalue can be obtained by substituting the fixed point into Eq. (20)

$$
\mu_{2}=g^{\prime}\left(\dot{x}_{1} *\right)=(1-b)^{4}
$$

If $0<b<2,\left|\mu_{2}\right|<1$. In this condition, $\mu_{1}=1,\left|\mu_{2}\right|<1$, therefore the solution is asymptotically stable. It is noticed that the stability is determined only by $b$ which is related to the property of the two objects in collision. In other words, the collision position and external force have nothing to do with the stability.

By solving the equations of step 1, 3, 4, 5, 7, 8 in Appendix A, the intervals of these steps can be obtained

$$
\begin{aligned}
& t_{1}=t_{0}+\frac{1}{\omega_{n}} \cos ^{-1}\left(-\frac{a_{1}}{h_{0}}\right) \\
& t_{2}=t_{1}+\frac{1}{\omega_{n}}\left(\tan ^{-1} \frac{\dot{x}_{1}^{+}}{\omega_{n}\left(a_{1}-\frac{f}{\omega_{n}^{2}}\right)}+\cos ^{-1}\left(\frac{\frac{f}{\omega_{n}^{2}}-a_{2}}{\left.\left.\sqrt{\left(a_{1}-\frac{f}{\omega_{n}^{2}}\right)^{2}+\left(\frac{\dot{x}_{1}^{+}}{\omega_{n}}\right)^{2}}\right)\right)}\right.\right. \\
& \left.t_{3}=t_{2}+\frac{1}{\omega_{n}} \tan ^{-1} \frac{\dot{x}_{2}}{\omega_{n} a_{2}}\right) \\
& t_{4}=t_{3}+\cos ^{-1}\left(\frac{a_{3}}{\sqrt{a_{2}^{2}+\left(\frac{\dot{x}_{2}}{\omega_{n}}\right)^{2}}}\right) \\
& t_{5}=t_{4}+\tan ^{-1} \frac{\dot{x}_{4}^{+}}{\omega_{n}\left(a_{3}+\frac{f}{\omega_{n}^{2}}\right)^{2}}-\frac{1}{\omega_{n}} \cos ^{-1}\left(\frac{a_{4}+\frac{f}{\omega_{n}^{2}}}{h_{3}}\right)
\end{aligned}
$$


Therefore we can get the period

$$
\begin{aligned}
T(k)=\frac{1}{\omega_{n}} & {\left[\cos ^{-1} \frac{f / \omega_{n}^{2}-a_{2}}{\sqrt{\left(a_{1}-f / \omega_{n}^{2}\right)^{2}+\left(\dot{x}_{1}^{+}(k) / \omega_{n}\right)^{2}}}-\cos ^{-1} \frac{f / \omega_{n}^{2}-a_{1}}{\sqrt{\left(a_{1}-f / \omega_{n}^{2}\right)^{2}+\left(\dot{x}_{1}^{+}(k) / \omega_{n}\right)^{2}}}\right.} \\
& +\cos ^{-1} \frac{a_{2}}{\sqrt{a_{2}^{2}+\left(\dot{x}_{2}^{+}(k) / \omega_{n}\right)^{2}}}+\cos ^{-1} \frac{a_{3}}{\sqrt{a_{2}^{2}+\left(\dot{x}_{2}^{+}(k) / \omega_{n}\right)^{2}}} \\
& +\cos ^{-1} \frac{f / \omega_{n}^{2}+a_{4}}{\sqrt{\left(a_{3}+f / \omega_{n}^{2}\right)^{2}+\left(\dot{x}_{4}^{+}(k) / \omega_{n}\right)^{2}}}-\cos ^{-1} \frac{f / \omega_{n}^{2}+a_{3}}{\sqrt{\left(a_{3}+f / \omega_{n}^{2}\right)^{2}+\left(\dot{x}_{4}^{+}(k) / \omega_{n}\right)^{2}}} \\
& \left.+\cos ^{-1} \frac{a_{4}}{\sqrt{a_{4}^{2}+\left(\dot{x}_{5}^{+}(k) / \omega_{n}\right)^{2}}}+\cos ^{-1} \frac{a_{1}}{\sqrt{a_{4}^{2}+\left(\dot{x}_{5}^{+}(k) / \omega_{n}\right)^{2}}}\right]
\end{aligned}
$$

The perturbation of period caused by the impact in $x=a_{1}$ is,

$$
\Delta T_{1}=\frac{1}{\omega_{n}}\left(\tan ^{-1}\left(\frac{\dot{x}_{1}^{+}}{a_{1} \omega_{n}}\right)-\tan ^{-1}\left(\frac{\dot{x}_{1}}{a_{1} \omega_{n}}\right)\right)=-\frac{1}{\omega_{n}} \tan ^{-1}\left(\frac{a_{1} \omega_{n} b \dot{x}_{1}^{+}}{a_{1}^{2} \omega_{n}^{2}+(1-b)\left(\dot{x}_{1}^{+}\right)^{2}}\right)
$$

The perturbation of period caused by the torque between $a_{1}$ and $a_{2}$ is,

$$
\begin{aligned}
\Delta T_{2}= & \frac{1}{\omega_{n}}\left[\cos ^{-1} \frac{f / \omega_{n}^{2}-a_{2}}{\sqrt{\left(a_{1}-f / \omega_{n}^{2}\right)^{2}+\left(\dot{x}_{1}^{+} / \omega_{n}\right)^{2}}}-\cos ^{-1} \frac{f / \omega_{n}^{2}-a_{1}}{\sqrt{\left(a_{1}-f / \omega_{n}^{2}\right)^{2}+\left(\dot{x}_{1}^{+} / \omega_{n}\right)^{2}}}\right] \\
& -\frac{1}{\omega_{n}}\left[\tan ^{-1}\left(\frac{\sqrt{\left(\dot{x}_{1}^{+} / \omega_{n}\right)^{2}+a_{1}^{2}-a_{2}^{2}-2\left(a_{1}-a_{2}\right) f / \omega_{n}^{2}}}{a_{2}}\right)-\tan ^{-1}\left(\frac{\dot{x}_{1}^{+}}{a_{1} \omega_{n}}\right)\right]
\end{aligned}
$$

\section{Simulation results}

Based on the equations above, simulation of a simplified model for the escapement system has been conducted. Assume the mass $M=1 \mathrm{~kg}$, the spring constant $K=1 \mathrm{~N} / \mathrm{m}, b=0.1, a_{1}=0.1 \mathrm{~m}, a_{2}=0.05$. The following diagrams show the simulation results for different parameters. Figure 3 is the Poincare map for two different initial conditions, one with $1 \mathrm{~N}$ external force and $1 \mathrm{~m}$ initial amplitude, the other with $0.5 \mathrm{~N}$ external force and $3 \mathrm{~m}$ initial amplitude. The velocity before impact at position $a_{1}$, the period and the amplitude for each cycle are studied and compared.

From the result, it is noted that both motions are convergent. It is because the criteria for convergence are satisfied. From the above section, we know that the stability is only related to $b$. The result shows that the external force and initial amplitude have no effect on the convergence. It is also noted that the period for both motions are identical as $6.2832 \mathrm{~s}$, which is the natural frequency of the system. That means, the initial condition for this system has nothing to do with the period. It can be found that stable velocities are different. This is because it is mainly determined by the external force. Although condition (a) has smaller velocity at the beginning, which is because the initial amplitude is small, the converged velocity is greater than that of condition (b). For the amplitude, the situation is the same. The converged amplitude is also determined by the external force. Therefore, the system with greater force has greater amplitude. It is also found that the converging speed is different. That is also dependent on the external force. From above, we can find that the Poincare map is convenient to analyze the influence of the initial condition on convergence properties. 

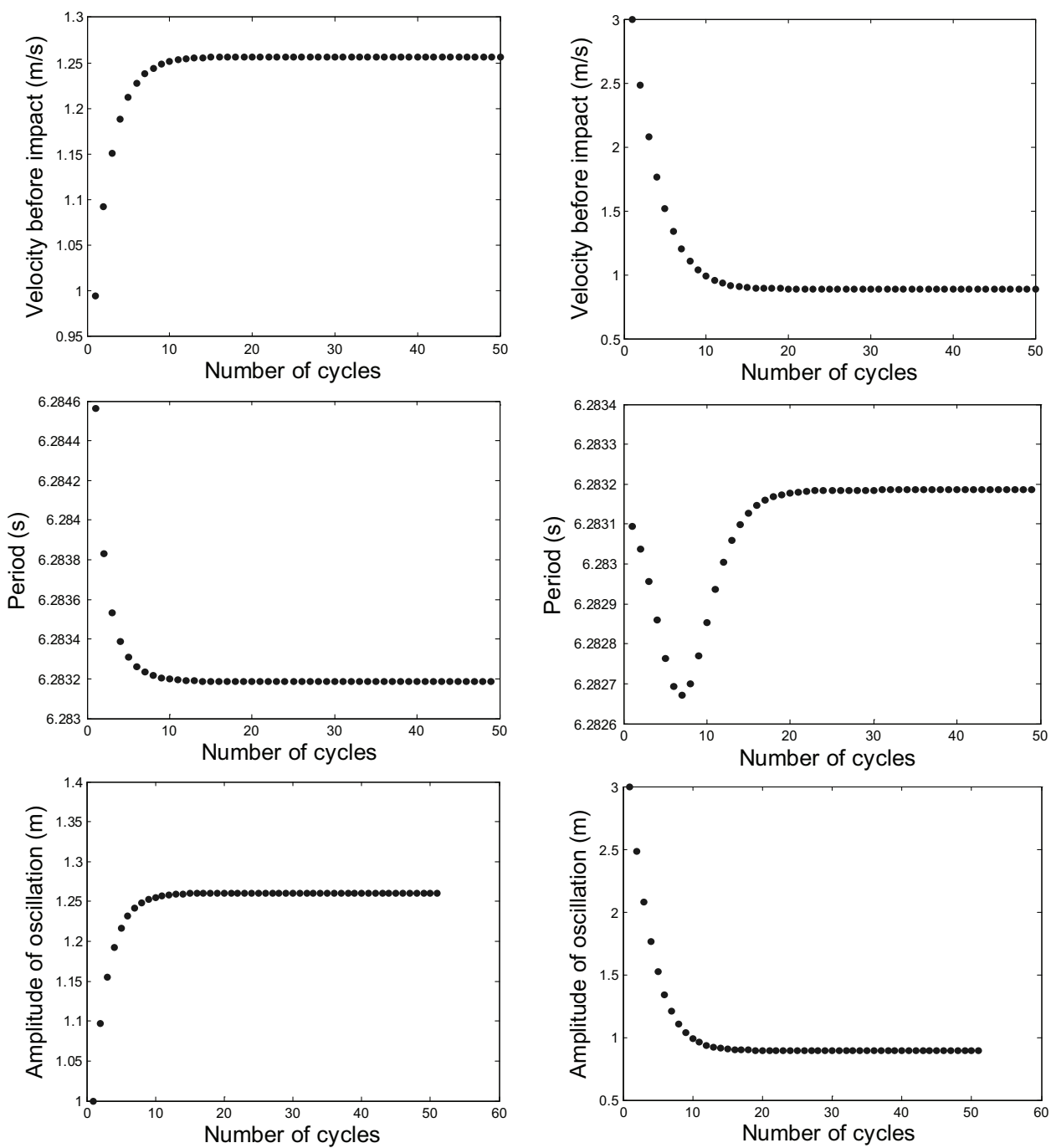

(a) $\mathrm{F}=1, \mathrm{x}(0)=1$

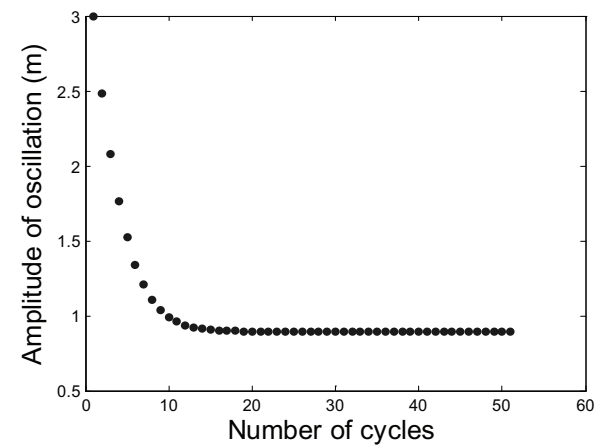

(b) $\mathrm{F}=0.5, \mathrm{x}(0)=3$

Fig. 3. Poincare map.
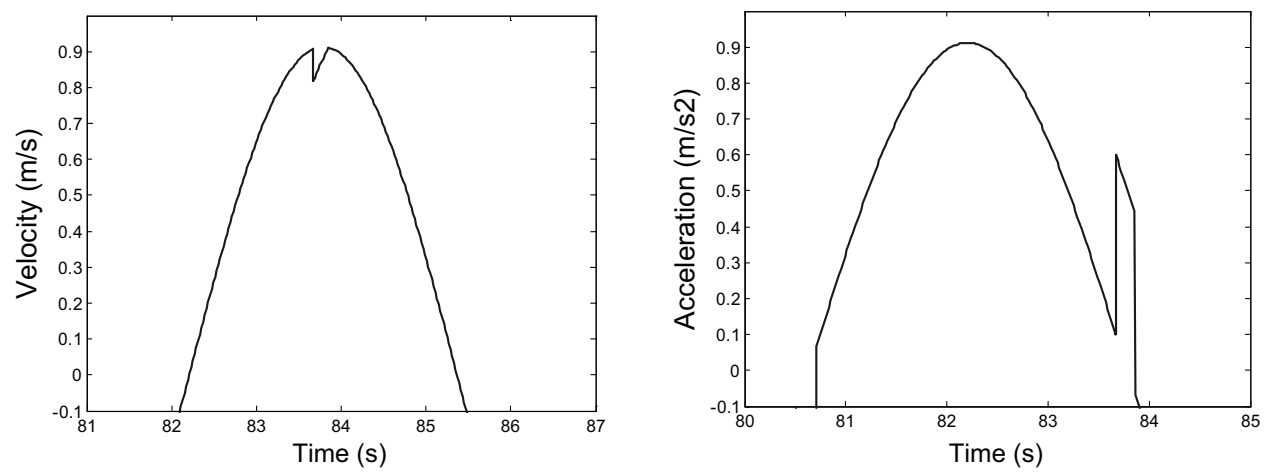

Fig. 4. Shock details. 


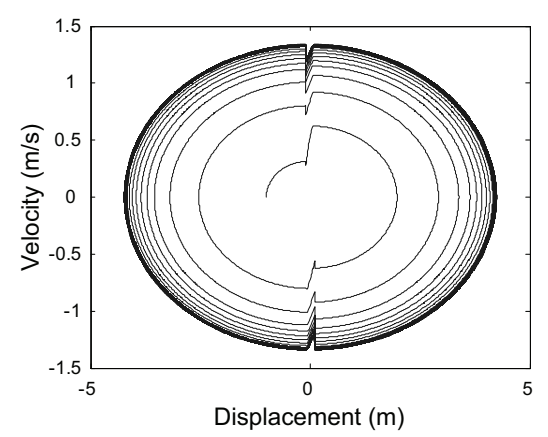

(a) $\mathrm{K}=0.1$

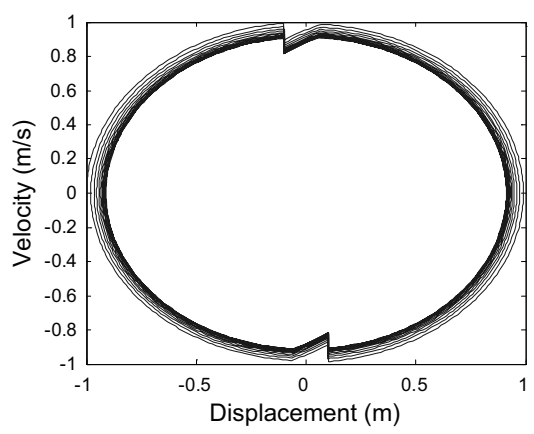

(b) $\mathrm{K}=1$

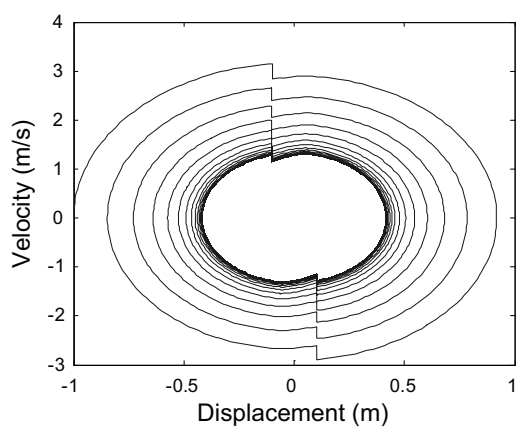

(c) $\mathrm{K}=10$

Fig. 5. Phase diagram.

Figure 4 shows the neighborhood where the impact occurs in time history. From the graph, the details of the velocity and acceleration change caused by the collision can be easily observed. Moreover, the impact force and the consumed energy can be evaluated from the graph.

Figure 5 is the phase diagram for three different spring constants, $K=0.1 \mathrm{~N} / \mathrm{m}, K=1 \mathrm{~N} / \mathrm{m}$ and $K=10 \mathrm{~N} / \mathrm{m}$. It can be found that each system converges to a limit cycle. The dimensions of the limit cycles are different. The smaller the spring constant, the greater the converged amplitude. The greater spring constant, the smaller maximum velocity. Impact is also clearly shown. Small spring constant can recover the velocity drop caused by collision faster than big spring constant.

\section{Conclusions}

In previous section, a simplied model is developed to describe the escapement mechanism in mechanical watch movement. The impulsive differential equation is applied to study the dynamics of the periodically forced oscillation system with impact. Poincare map is adopted to investigate the convergence of the system. When they are used together, the nonlinear behavior and stability of oscillating motion can be studied conveniently. First the motion is described in eight different steps. After combining the system equations into one single impulsive differential equation, the complicated system is therefore well defined. The existense and uniqueness of the solution can be checked by the initial condition. By deriving the Poincare map of the state of certain condition, the eigenvalue can be found by substituting the fixed point into the derivative of the map function. The stability can therefore be studied. Under the assumption $a_{1}=-a_{3}, a_{2}=-a_{4}$, the convergence is only related to $b$. However, if this assumption does not hold, the convergence will be dependent on the external force and applied position. The simulation shows and verifies the effect of different parameters. The result indicates that the model can describe the system properly.

\section{Appendix A: The model of the first order impact system}

\section{First half period}

Step1: The mass starts moving under the restoring force of the spring. The equation of the system is

$$
\ddot{x}+k x=0
$$

Step2: Impact occurs at the spot of $a_{1}$ and instant $t_{1}$, the impulse equation is

$$
\dot{x}\left(t_{1}^{+}\right)=\dot{x}_{1}^{+}=(1-b) \dot{x}_{1}
$$


Step3: The system continues moving under the external force $F$. The system equation is

$$
\ddot{x}+k x=f
$$

Step4: The external force stops functioning. The system remains the sinusoidal oscillation with the identical system Eq. (A-1) as Step 1.

\section{Second half period}

Step5: It is similar with the step 1 in the first half period. The system equation is identical as Eq. (A-1).

Step6: Impact occurs at the spot of $a_{3}$ and instant $t_{4}$, the impulse equation is

$$
\dot{x}\left(t_{4}^{+}\right)=\dot{x}_{4}^{+}=(1-b) \dot{x}_{4}
$$

Step7: The system equation is

$$
\ddot{x}+k x=-f
$$

Step8: The system equation is the same as Eq. (A-1)

\section{References}

[1] Y. Fu, A study on the dynamics of periodical impact mechanism with an application in mechanical watch escapement, The Chinese University of Hong Kong, 2008.

[2] D. Stewart, Rigid-body dynamics with friction and impact, SIAM Review 42(1) (2000), 3-39.

[3] K. Popp, Non-smooth mechanical systems, Journal of Applied Mathematics and Mechanics 64(5) (2000), 765-772.

[4] B. Brogliato, Nonsmooth mechanics: Models, dynamics and control, London, Springer, 1999.

[5] B. Brogliato, S. Niculescu and P. Orhant, On the control of finite-dimensional mechanical systems with unilateral constraints, IEEE Transactions on Automatic Control 42(2) (1997), 200-215.

[6] R. Leine and D. Campen, Discontinuous bifurcations of periodic solutions, Mathematical and Computer Modelling 36 (2002), $259-273$.

[7] J. Guckenheimer and P. Holmes, Nonlinear oscillations, dynamical systems and bifurcations of vector fields, Berlin, Springer-Verlag, 1983.

[8] A. Fidlin, Nonlinear oscillations in mechanical engineering, Berlin, Springer, 2006

[9] S. Shaw and P. Holmes, A periodically forced piecewise linear oscillator, Journal of Sound and Vibration 90(1) (1983), 129-155.

[10] F. Peterka, Dynamics of double impact oscillators, Facta Universitatis 2(10) (2000), 1177-1190.

[11] O. Janin and C. Lamarque, Stability of singular periodic motions in a vibro-impact oscillator, Nonlinear Dynamics 28 (2002), $231-241$.

[12] W. Zhang, W. Zhang and K. Turner, Nonlinear dynamics of micro impact oscillators in high frequency MEMS switch application, The 13th International Conference on Solid-State Sensors, Actuators and Microsystems Seoul, Korea, 2005.

[13] A. Roup and D. Bernstein, On the dynamics of the escapement mechanism of a mechanical clock, Proceedings of the 38th IEEE Conference on Decision and Control (1999).

[14] A. Roup et al., Limit cycle analysis of the verge and foliot clock escapement using impulsive differential equations and poincare maps, Int J Control 76(17) (2003), 1685-1698.

[15] M. Senator, Synchronization of two coupled escapement-driven pendulum clocks, Journal of Sound and Vibration 291(3-5) (2006), 566603.

[16] V. Lakshmikantham, D. Bainov and P. Simeonov, Theory of impulsive differential equations, World Scientific, 1988.

[17] D.D. Bainov and P.S. Simeonov, Impulsive differential equations: Asymptotic properties of the solutions, Singapore, World Scientific, 1995. 

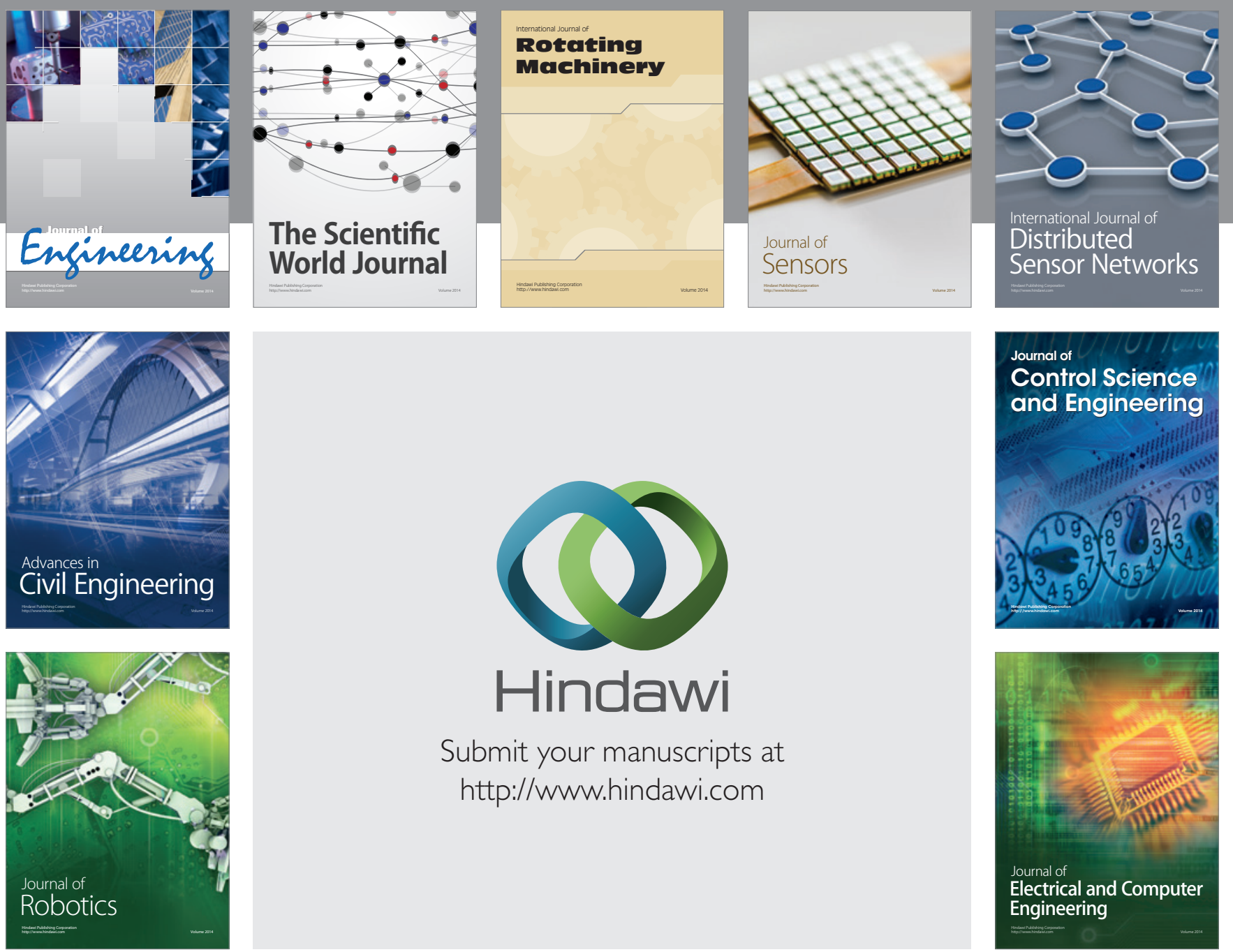

Submit your manuscripts at

http://www.hindawi.com
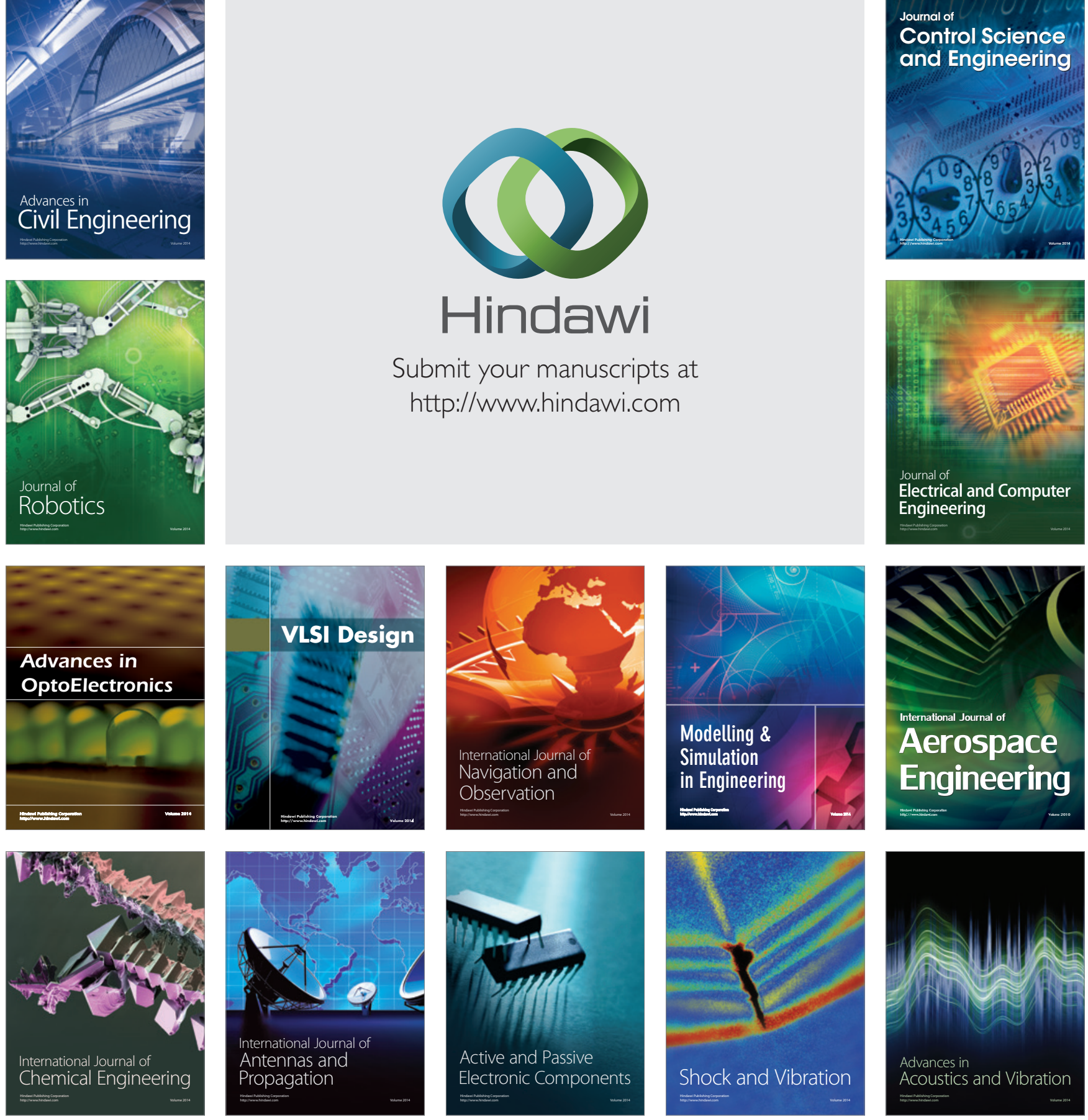